\title{
Bounds for the Second Hankel Determinant of Certain Univalent Functions
}

\author{
Renuka Devi K, Hamid Shamsan, S. Latha
}

\begin{abstract}
We study the estimates for the Second Hankel determinant of analytic functions. Our class includes (j,k)-convex, (j,k)-starlike functions and Ma-Minda starlike and convex functions..
\end{abstract}

Index Terms: Hankel determinant, starlike functions, Ma-Minda starlike and convex functions.

\section{INTRODUCTION}

Let A denote the class of functions of form

$$
f(z)=z+\sum_{n=2}^{\infty} a_{n} z^{n},(1.1)
$$

which are analytic in the open unit disk $\mathrm{U}=\{z: z \in \mathrm{C}$ and $|z|$ $<1\}$, and $\mathrm{S}$ denote the

subclass of A consisting of all function which are univalent in $\mathrm{U}$.

We denote by $\mathcal{S}^{*}, \mathcal{K}, \mathcal{S}_{s}^{*}, \mathcal{K}_{s}$ the familiar subclasses consisting of functions which, respec-

tively, starlike, convex, starlike with respect to symmetric points and convex with respect to symmetric points in U.

In 1976, Noonan and Thomas [13] stated the $q^{\text {th }}$ Hankel determinant of $f(z)$ for $q \geq 1$ and $n \geq 1$ as

$\left|\begin{array}{cccc}a_{n} & a_{n+1} & \ldots & a_{n+q-1} \\ a_{n+1} & \ldots & \ldots & \ldots \\ \ldots & \ldots & \ldots & \ldots \\ a_{n+q-1} & \ldots & \ldots & a_{n+2 q-2}\end{array}\right|, a_{1}=1$

This determinant has also been considered by several authors. For example Noor in [12] determined the rate of growth $H_{q}(k)$ as $k \rightarrow \infty$ for functions $f$ given by (1.1) with bounded boundary. Ehrenborg in [2] studied the Hankel determinant of exponential polynomials. The Hankel transform of an integer sequence and some of its properties were discussed by Hayman in [5].

Easily, one can observe that the Fekete and Szeg" $o$ functional is $H_{2}(1)$. Fekete and Szeg" $o$ [3]

then further generalised the estimate $\left|a_{3}-\mu a_{2}^{2}\right|_{\text {where }} \mu$ is real and $f \in \mathrm{S}$.

For our discussion in this paper, we consider the Hankel

Revised Manuscript Received on July 08, 2019

RENUKA DEVI K, Department of Mathematics, Yuvaraja's College, University of Mysore,Mysuru-570 005, INDIA. amst1920@gmail.com HAMID SHAMSAN, Department of Mathematics, Yuvaraja's College, University of Mysore,Mysuru-570 005, INDIA.

S. LATHA, Department of Mathematics, Yuvaraja's College,

University of Mysore,Mysuru-570 005, INDIA determinant th the case of $q=2$ and $n=2$, known as second Hankel determinant:

$H_{2}(2)=\left|\begin{array}{ll}a_{2} & a_{3} \\ a_{3} & a_{4}\end{array}\right|=\left|a_{2} a_{4}-a_{3}^{2}\right|$

and obtain an upper bound to $H_{2}(2)$ for $f(z) \in \mathrm{S}^{j, k}$ and $f(z) \in$ $\mathrm{K}^{j, k}$. Janteng et al. [7] have considered the functional $\left|H_{2}(2)\right|$ and found a sharp bound, the subclass of $\mathrm{S}$ as $\left\langle\left\{f^{0}(z)\right\}>0\right.$. In their work, they have shown that if $f \in \mathrm{S}$, then $\left|H_{2}(2)\right| \leq 4 / 9$. These authors [6, 7] also studied the second Hankel determinant and sharp bound for the classes of starlike and convex functions, close-to-starlike and close-to-convex functions with respect to symmetric points have shown that $\left|H_{2}(2)\right| \leq 1,\left|H_{2}(2)\right| \leq 1 / 8,\left|H_{2}(2)\right| \leq 1,\left|H_{2}(2)\right| \leq 1 / 9$,

respectively.

Definition 1.1. Let $k$ be a positive integer. A domain $\mathrm{D}$ is said to be $k$-fold symmetric if a rotation of $\mathrm{D}$ about the origin through an angle ${ }^{2} \frac{\pi}{k}$ carries $\mathrm{D}$ onto itself. A function $f$ is said to be $k$-fold symmetric in $\mathrm{U}$ iffor every $z$ in $\mathrm{U}$

\section{1. $2 \pi i 2 \pi i$}

$f(e k z)=e k f(z)$.

The family of all k-fold symmetric functions is denoted by $\mathrm{S}^{k}$ and for $k=2$ we get class of the odd univalent functions.

The notion of $(j, k)$-symmetrical functions $(k=2,3, \ldots ; j=$ $0,1,2, \ldots, k-1)$ is a generalization of the notion of even, odd, $k$-symmetrical functions and also generalize the well-known result that each function defined on a symmetrical subset can be uniquely expressed as the sum of an even function and an odd function.

The theory of $(j, k)$ symmetrical functions has many interesting applications, for instance in the investigation of the set of fixed points of mappings, for the estimation of the absolute value of some integrals, and for obtaining some results of the type of Cartan uniqueness theorem for holomorphic mappings [11].

Definition 1.2. Let $\varepsilon=\left(e^{\frac{2 \pi i}{k}}\right)$ and $j=0,1,2, . ., k-1$ where $k$ $\geq 2$ is a natural number. $A$

function $f: \mathrm{U} 7 \rightarrow \mathrm{C}$ is called $(j, k)$-symmetrical if $f(\varepsilon z)=\varepsilon^{j} f(z), z \in \mathrm{U}$.

We note that the family of starlike functions with respect to $(j, k)$-symmetric points is denoted be $S^{(j, k)}$. Also, $S^{(0,2)}, S^{(1,2)}$ and $\mathbf{S}^{(1, k)}$ are called even, odd and $k$-symmetric functions respectively. We have the following decomposition theorem. Definition 1.3. A function $f(z) \in \mathrm{A}$ is in the class $\mathrm{S}_{j, k}{ }^{*}$ if $\Re\left\{\frac{z f^{\prime}(z)}{f_{j, k}(z)}\right\}>0$ where $f_{j, k}$ defined by (1.2). 
Definition 1.4. A function $f(z) \in \mathrm{A}$ is in the class $\mathrm{K}_{j, k}$ if $\Re\left\{\frac{\left(z f^{\prime}(z)\right)^{\prime}}{f_{j, k}^{\prime}(z)}\right\}>0$

where $f_{j, k}$ defined by (1.2).

Theorem 1.5. [11] For every mapping $f: \mathrm{D} 7 \rightarrow \mathrm{C}$, and $\mathrm{D}$ is a $k$-fold symmetric set, there

exists exactly the sequence of $(j, k)$ - symmetrical functions $f_{j, k}$, $k-1$

$f(z)={ }^{\mathrm{X}} f_{j, k}(z)$

$j=0$

where

$$
f_{j, k}(z)=\frac{1}{k} \sum_{v=0}^{k-1} \varepsilon^{-v j} f\left(\varepsilon^{v} z\right),
$$

$(f \in \mathcal{A} ; k=1,2, \ldots ; j=0,1,2, \ldots, k-1)$

From (1.2) we can get

$$
f_{j, k}(z)=\frac{1}{k} \sum_{v=0}^{k-1} \varepsilon^{-v j} f\left(\varepsilon^{v} z\right)=\frac{1}{k} \sum_{v=0}^{k-1} \varepsilon^{-v j}\left(\sum_{n=1}^{\infty} a_{n}\left(\varepsilon^{v} z\right)^{n}\right),
$$

then

$$
\begin{aligned}
f_{j, k}(z)= & \sum_{n=1}^{\infty} \delta_{n, j} a_{n} z^{n}, a_{1}=1 \\
\psi & 1 \sum^{k-1} \varepsilon^{(n-j) v}= \begin{cases}1, & n=l k+j\end{cases}
\end{aligned}
$$

$n=-, \quad(1.3) k$

$v=0$ ? $0, n 6=l k+j$;

Definition 1.6.[1] Let $\mathrm{P}$ denote the class of analytic functions $p: \mathrm{U} \rightarrow \mathrm{C}, p(0)=1$,

and $\left\langle\{p(z)\}>0\right.$, then $p(z) \prec \frac{1+z}{1-z \text {. }}$

The class $\mathrm{P}$ can be completely characterized in terms of subordination.

We need the following lemmas to derive our results.

Lemma 1.7. [1] If the function $p \in \mathrm{P}$ is given by the series

$$
p(z)=1+c_{1} z+c_{2} z^{2}+c_{3} z^{3}+\ldots, \quad(1.4)
$$

then the following sharp estimate holds:

$$
\left|c_{n}\right| \leq 2(n=1,2, \ldots) .
$$

Lemma 1.8. [4] If the function $p \in \mathrm{P}$ is given by the series (1.4), then

$$
\begin{aligned}
& 2 c_{2}=c_{1}^{2}+x\left(4-c_{1}^{2}\right),(1.5) \\
& 4 c_{3}=c_{1}^{3}+2\left(4-c_{1}^{2}\right) c_{1} x-c_{1}\left(4-c_{1}^{2}\right) x^{2}+2\left(4-c_{1}^{2}\right)\left(1-|x|^{2}\right) z
\end{aligned}
$$

for some $x, z$ with $|x| \leq 1$ and $|z| \leq 1$.

\section{MAIN RESULTS}

Definition 2.1. Let $\phi: U \rightarrow \mathrm{C}$ be analytic, and let the Maclaurin series of $\phi$ is given by

$$
\phi(z)=1+D_{1} z+D_{2} z^{2}+D_{3} z^{3}+\ldots \quad\left(D_{1}, D_{2} \in \mathrm{R}, D_{1}>0\right) .
$$

The class $S^{*(j, k)}(\phi)$ of starlike functions with respect to $\phi$ consists of functions $f \in \mathrm{A}$ satisfying the subordination $\frac{z f^{\prime}(z)}{f_{j, k}(z)} \prec \varphi(z)$.

Theorem 2.2. Let the function $f \in S^{*}(\phi)$ be given by (1.1)

2. If $D_{1}, D_{2}$ and $D_{3}$ satisfy the conditions

$$
\beta D_{1}^{2}+2 \alpha\left|D_{2}\right|+\gamma D_{1} \leq 0
$$

$\left|\psi_{1}\left(3-\psi_{3}\right)^{2} D_{1} D_{3}+\delta D_{1}^{4}+\beta D_{1}^{2} D_{2}-\psi_{1}^{2}\left(4-\psi_{4}\right)\left(2-\psi_{2}\right) D_{2}^{2}\right|-(\gamma-\lambda) D_{1}^{2} \leq 0$,

then the second Hankel determinant satisfies

$\left|a_{2} a_{4}-a_{3}^{2}\right| \leq \frac{D_{1}^{2} \psi_{1}^{2}}{\left(3-\psi_{3}\right)^{2}}$.

3. If $D_{1}, D_{2}$ and $D_{3}$ satisfy the conditions

$\beta D_{1}^{2}+2 \alpha\left|D_{2}\right|+\gamma D_{1} \geq 0$

$\left|\psi_{1}\left(3-\psi_{3}\right)^{2} D_{1} D_{3}-\psi_{1}^{2}\left(4-\psi_{4}\right)\left(2-\psi_{2}\right) D_{2}^{2}+\delta D_{1}^{4}+\beta D_{1}^{2} D_{2}\right|-\alpha D_{1}\left|D_{2}\right|-$

$\frac{1}{2} \beta D_{1}^{3}-\frac{1}{2} \lambda D_{1}^{2} \geq 0$,

or the conditions

$$
\begin{aligned}
& \beta D_{1}^{2}+2 \alpha\left|D_{2}\right|+\gamma D_{1} \leq 0 \\
& \left|\psi_{1}\left(3-\psi_{3}\right)^{2} D_{1} D_{3}+\delta D_{1}^{4}+\beta D_{1}^{2} D_{2}-\psi_{1}^{2}\left(4-\psi_{4}\right)\left(2-\psi_{2}\right) D_{2}^{2}\right|-(\gamma-\lambda) D_{1}^{2} \geq 0,
\end{aligned}
$$

then the second Hankel determinant satisfies

$$
\left|a_{2} a_{4}-a_{3}^{2}\right| \leq \frac{1}{\left(4-\psi_{4}\right)\left(3-\psi_{3}\right)^{2}\left(2-\psi_{2}\right)}\left|\psi_{1}\left(3-\psi_{3}\right)^{2} D_{1} D_{3}-\psi_{1}^{2}\left(4-\psi_{4}\right)\left(2-\psi_{2}\right) D_{2}^{2}+\delta D_{1}^{4}+\beta D_{1}^{2} D_{2}\right|
$$

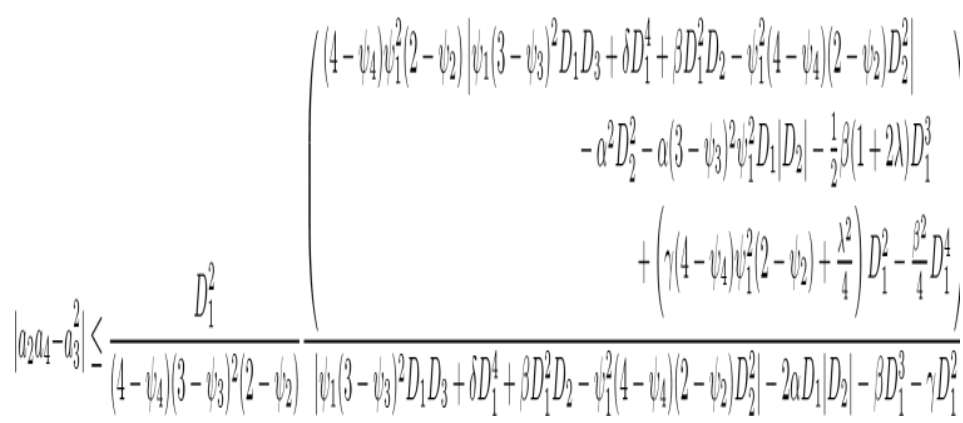

4. If $D_{1}, D_{2}$ and $D_{3}$ satisfy the

$\beta D_{1}^{2}+2 \alpha\left|D_{2}\right|+\gamma D_{1}>0$

$\left|\psi_{1}\left(3-U_{3}\right)^{2} D_{1} D_{3}-\psi_{1}^{2}\left(4-\psi_{4}\right)\left(2-\psi_{2}\right) D_{2}^{2}+\delta D_{1}^{4}+\beta D_{1}^{2} D_{2}\right|-\alpha D_{1}\left|D_{2}\right|-$

$\frac{1}{2} \beta D_{1}^{3}-\frac{1}{2} \lambda D_{1}^{2} \geq 0$,

then the second Hankel determinant satisfies where

$$
\begin{aligned}
\alpha & =\psi_{1}\left(3-\psi_{3}\right)^{2}-\psi_{1}^{2}\left(4-\psi_{4}\right)\left(2-\psi_{2}\right), \quad \beta=\psi_{1}^{2}\left(\frac{\left(2 \psi_{3}+3 \psi_{2}-2 \psi_{1} \psi_{2}\right)\left(3-\psi_{3}\right)-2 \psi_{2}\left(4-\psi_{4}\right)\left(2-\psi_{2}\right)}{\left(2-\psi_{2}\right)}\right) \\
\gamma & =\psi_{1}^{2}\left(3-\psi_{3}\right)^{2}-\psi_{1}^{2}\left(4-\psi_{4}\right)\left(2-\psi_{2}\right) \\
\delta & =\frac{\psi_{1}^{2} \psi_{2} \psi_{3}\left(3-\psi_{3}\right)\left(2-\psi_{2}\right)-\psi_{1}^{2} \psi_{2}\left(4-\psi_{4}\right)}{\left(2-\psi_{2}\right)} \text { and } \\
\lambda & =\psi_{1}^{2}\left(3-\psi_{3}\right)^{2}-2 \psi_{1}^{2}\left(4-\psi_{4}\right)\left(2-\psi_{2}\right) .
\end{aligned}
$$

Proof. Since $f \in S^{*}(j, k)(\phi)$, there exists an analytic function $w$ with $w(0)=0$ and $|w(z)|<1$ in $\mathrm{U}$ such that

$$
\frac{z f^{\prime}(z)}{f_{j, k}(z)}=\varphi(w(z))
$$

Define the function $p_{1}$ by

$$
p_{1}(z)=\frac{1+w(z)}{1-w(z)}=1+c_{1} z+c_{2} z^{2}+\ldots,
$$

or, equivantely

$$
w(z)=\frac{p_{1}(z)-1}{p_{1}(z)+1}=\frac{1}{2}\left(c_{1} z+\left(c_{2}-\frac{c_{1}^{2}}{2}\right) z^{2}+\left(c_{3}-c_{1} c_{2}+\frac{c_{1}^{3}}{4}\right) z^{3}+\ldots\right)
$$

Then $p_{1}$ is analytic in $\mathrm{U}$ with $p_{1}(0)=0$ and has a positive real part in U. By using(2.4) together with (2.1), it is evident that

$$
\varphi\left(\frac{p_{1}(z)-1}{p_{1}(z)+1}\right)=1+\frac{1}{2} D_{1} c_{1} z+\left(\frac{1}{2} D_{1}\left(c_{2}-\frac{c_{1}^{2}}{2}\right)+\frac{1}{4} D_{2} c_{1}^{2}\right) z^{2}+\ldots
$$

By (2.3) we have 


$$
\frac{z f^{\prime}(z)}{f_{j, k}(z)}=1+\frac{1}{2} D_{1} c_{1} z+\left(\frac{1}{2} D_{1}\left(c_{2}-\frac{c_{1}^{2}}{2}\right)+\frac{1}{4} D_{2} c_{1}^{2}\right) z^{2}+\ldots,
$$

so that

$$
\left(\frac{z+2 a_{2} z^{2}+3 a_{3} z^{3}+\ldots}{\psi_{1} z+\psi_{2} a_{2} z^{2}+\psi_{3} a_{3} z^{3}+\ldots}\right)=1+\frac{1}{2} D_{1} c_{1} z+\left(\frac{1}{2} D_{1}\left(c_{2}-\frac{c_{1}^{2}}{2}\right)+\frac{1}{4} D_{2} c_{1}^{2}\right) z^{2}+\ldots
$$

which implies the equality

$$
z+2 a_{2} z^{2}+3 a_{3} z^{3}+\ldots=v_{1} z+\left(v_{2} a_{2}+\frac{1}{2} D_{1} c_{1} v_{1}\right) z^{2}+\left(v_{3} a_{3}+\frac{1}{2} v_{2} D_{1} C_{1} a_{2}+\frac{1}{2} D_{1} v_{1}\left(c_{2}-\frac{1}{2}\right)+-D_{2} v_{1} c_{1}^{2}\right) z^{3}+\ldots .
$$

Equating the coefficients on both sides we have $a_{2}=\frac{D_{1}\left(1 y_{1} y_{1}\right.}{2\left(2-v_{2}\right)^{\prime}}$

$a_{3}=\frac{1}{\left(3-v_{3}\right)}\left(\left(\frac{\left(D_{1}^{2} \psi_{1} \psi_{2}\right.}{\left(2-v_{2}\right)}-D_{1} \psi_{1}+D_{2} \psi_{1}\right) c_{1}^{2}+2 D_{1} \psi_{1} C_{2}\right)$,

$a_{4}=\frac{1}{8\left(1-v_{4}\right)\left(3-v_{3}\right)\left(2-v_{2}\right)}\left[\left(-2\left(3-v_{3}\right)\left(2-v_{2}\right) D_{2}+v_{1}\left(3-v_{3}\right)\left(2-v_{2}\right) D_{1}+v_{1} v_{2} v_{3} D_{1}^{3}-\left(v_{1} v_{3}\left(2-v_{2}\right)\right)+\right.\right.$

\section{$v_{1} v_{2}\left(3-v_{3}\right) D_{1}^{2}+\left(v_{1} v_{3}\left(2-v_{2}\right)+v_{1} v_{2}\left(3-v_{3}\right)\right) D_{1} D_{2}+\left(2-v_{2}\right)\left(3-v_{3}\right) c_{1}^{3}+4 v_{1}\left(2-v_{2}\right)\left(3-v_{3}\right) B_{1} c_{3}$}

\section{$-2\left(\left(v_{1} v_{3}\left(2-v_{2}\right)+v_{1} v_{2}\left(3-v_{3}\right) D_{1}^{2}-\left(2 v_{1}\left(2-v_{2}\right)\left(3-v_{3}\right) D_{1}+2\left(2-v_{2}\right)\left(3-v_{3}\right) D_{2}\right)\right) c_{1} c_{2}\right)$}

Therefore where $\alpha, \beta, \gamma, \delta$ and $\delta$ are given by (2.2).

Let

$$
\begin{gathered}
d_{1}=4\left(3-\psi_{3}\right) \psi_{1}^{2} D_{1}, \quad d_{2}=\frac{2}{\left(3-\psi_{3}\right)}\left(\beta D_{1}^{2}+2 a D_{2}-2 \gamma D_{1}\right), \quad d_{3}=-\frac{4 \psi_{1}^{2}\left(4-\psi_{4}\right)\left(2-\psi_{2}\right)}{\left(3-\psi_{3}\right)} D_{1}, \\
d_{4}=\frac{1}{\left(3-\psi_{3}\right)}\left(\delta D_{1}^{3}+\gamma D_{1}-2 a D_{2}+\psi_{2}\left(3-\psi_{3}\right)^{2} D_{3}-\psi_{1}^{2}\left(4-\psi_{4}\right)\left(2-\psi_{2}\right) \frac{D_{2}^{2}}{D_{1}}-\beta D_{1}^{2}+\beta D_{1} D_{2}\right)
\end{gathered}
$$

$$
\text { and } T=\frac{D_{1}}{16\left(4-\psi_{4}\right)\left(3-\psi_{3}\right)\left(2-\psi_{2}\right)} \text {. }
$$

Then

$$
\left|a_{2} a_{4}-a_{3}^{2}\right|=T\left|d_{1} c_{1} c_{3}+d_{2} c_{1}^{2} c_{2}+d_{3} c_{2}^{2}+d_{4} c_{1}^{4}\right| .
$$

Since the function $p\left(e^{i \theta} z\right)(\theta \in \mathrm{R})$ is in the class $\mathrm{P}$ for any $p \in$ $\mathrm{P}$, there is no loss of generality in assuming $c_{1}>0$. Write $c_{1}=$ $c, c \in[0,2]$. Substituting the values of $c_{2}$ and $c_{3}$ respectively from (1.5) and (1.6) in (2.8), we obtain

$$
\begin{aligned}
\left|a_{2} a_{4}-a_{3}^{2}\right|= & \frac{T}{4} \mid c^{4}\left(d_{1}+2 d_{2}+d_{3}+4 d_{4}\right)+2 x c^{2}\left(4-c^{2}\right)\left(d_{1}+d_{2}+d_{3}\right) \\
& +\left(4-c^{2}\right) x^{2}\left(-d_{1} c^{2}+d_{3}\left(4-c^{2}\right)\right)+2 d_{1} c\left(4-c^{2}\right)\left(1-|x|^{2} z\right) \mid .
\end{aligned}
$$

Replacing $|x|$ by $\mu$ and substituting the values of $d_{1}, d_{2}, d_{3}$ and $d_{4}$ from (2.7) yield $\left|a_{2} a_{4}-a_{3}^{2}\right| \leq \frac{T}{4}\left[\frac{c^{4}}{\left(3-\psi_{3}\right)}\left|4 \delta D_{1}^{3}+4\left(3-\psi_{3}\right)^{2} D_{3}-4 \psi_{1}^{2}\left(4-\psi_{4}\right)\left(2-\psi_{2}\right) \frac{D_{2}^{2}}{D_{1}}+4 \beta D_{1} D_{2}\right|+\frac{2 \mu c^{2}\left(4-c^{2}\right)}{\left(3-\psi_{3}\right)}\left(\alpha\left|D_{2}\right|+2 \beta D_{1}^{2}\right)\right.$

$$
\left.+\frac{4 \mu^{2}\left(4-c^{2}\right)}{\left(3-\psi_{3}\right)}\left(\lambda D_{1} c^{2}+16 \psi_{1}^{2}\left(4-\psi_{4}\right)\left(2-\psi_{2}\right) D_{1}\right)+8\left(3-\psi_{3}\right) \psi_{1}^{2} D_{1} c\left(4-c^{2}\right)\left(1-\mu^{2}\right)\right] .
$$

$=T\left[\frac{c^{4}}{4\left(3-v_{3}\right)}\left|4 B D_{1}^{3}+4\left(3-v_{3}\right)^{2} D_{3}-4 \psi_{1}^{2}\left(4-v_{4}\right)\left(2-v_{2}\right) \frac{D_{2}^{2}}{D_{1}}+4 B D_{1} D_{2}\right|+2\left(3-v_{3}\right) \psi_{1}^{2} D_{1} c\left(4-c^{2}\right)+\frac{\mu c^{2}\left(4-c^{2}\right)}{\left(3-v_{3}\right)}\right.$

$$
\left.\left(8 D_{1}^{2}+2 a \mid D_{2}\right)+\frac{\mu^{2}\left(4-c^{2}\right) D_{1}}{\left(3-v_{3}\right)}\left(\gamma^{2}-2\left(3-v_{3}\right)^{2} \psi_{1}^{2} c+4\left(4-\psi_{4}\right)\left(2-v_{2}\right) v_{1}^{2}\right)\right] .
$$

$\equiv F(c, \mu)$,

where $\alpha, \beta, \gamma, \delta$ and $\delta$ are given by (2.2).

Again, differentiating $F(c, \mu)$ in $(2.9)$ partially with respect to $\mu$ yields

$$
\frac{\partial F}{\partial \mu}=T\left[\frac{c^{2}}{\left(3-\psi_{3}\right)}\left(4-c^{2}\right)\left(\beta D_{1}^{2}+2 \alpha\left|D_{2}\right|\right)+\frac{2 \mu\left(4-c^{2}\right) D_{1}}{\left(3-\psi_{3}\right)}\left(\gamma c^{2}-2\left(3-\psi_{3}\right)^{2} \psi_{1}^{2} c+4\left(4-\psi_{4}\right)\left(2-\psi_{2}\right) \psi_{1}^{2}\right]\right.
$$

Then, for $0<\mu<1,0<q<1$ and for any fixed $c$ with $0<c$
$<2$, it is clear from (2.18) that $\frac{\partial F}{\partial \mu}>0$, that is, $F(c, \mu)$ is an increasing function of $\mu$. Hence, for fixed $c \in[0,2]$, the maximum of $F(c, \mu)$ occurs at $\mu=1$, and $\max F(c, \mu)=F(c, 1) \equiv G(c)$.

Also note that $G(c)=T\left[\frac{c^{4}}{\left(3-\psi_{3}\right)}\left(\left|4 \delta D_{1}^{3}+4 \beta D_{1} D_{2}+4\left(3-\psi_{3}\right)^{2} D_{3}-4 \psi_{1}^{2}\left(4-\psi_{4}\right)\left(2-\psi_{2}\right)\left(D_{2}^{2} / D_{1}\right)\right|-\beta D_{1}^{2}-2 \alpha\left|D_{2}\right|-\gamma D_{1}\right)\right.$

$$
\left.+\frac{4 c^{2}}{\left(3-\psi_{3}\right)}\left(\beta D_{1}^{2}+2 \alpha\left|D_{2}\right|+\lambda D_{1}\right)+16 \frac{\left(4-\psi_{4}\right)\left(2-\psi_{2}\right)}{\left(3-\psi_{3}\right)} \psi_{1}^{2} D_{1}\right]
$$

where $\lambda=\left(3-\psi_{3}\right)^{2} \psi_{1}^{2}-2\left(4-\psi_{4}\right)\left(2-\psi_{2}\right) \psi_{1}^{2}, \alpha, \beta, \gamma, \delta$ and $\delta$ are given by (2.2). Let

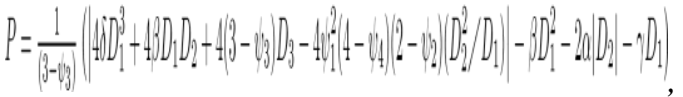

$$
\begin{aligned}
& Q=\frac{4}{\left(3-\psi_{3}\right)}\left(\beta D_{1}^{2}+2 \alpha\left|D_{2}\right|+\lambda D_{1}\right), \quad R=16 \frac{\psi_{1}^{2}\left(4-\psi_{4}\right)\left(2-\psi_{2}\right)}{\left(3-\psi_{3}\right)} D_{1}
\end{aligned}
$$

Since

$$
\begin{array}{cc}
R, & Q \leq 0, P \leq \frac{-Q}{4} ; \\
\max \left(P t^{2}+Q t+R\right)= \begin{cases}16 P+4 Q+R, & Q \geq 0, P \geq \frac{-Q}{8} \operatorname{or} Q \leq 0, P \geq \frac{-Q}{4}\end{cases} \\
\qquad \begin{array}{ll}
\frac{4 P R-Q^{2}}{4 P}, & Q>0, P \leq \frac{-Q}{8}
\end{array}
\end{array}
$$

where $0 \leq t \leq 4$.

Then we have

$$
\begin{aligned}
& \text { |R, } \quad Q \leq 0, P \leq \frac{-Y}{4} \text {; }
\end{aligned}
$$

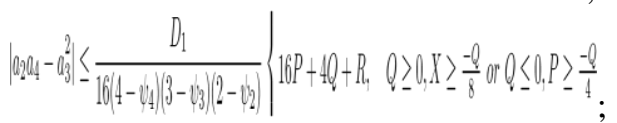

$$
\begin{aligned}
& \frac{4 P R-Q^{2}}{4 P}, \quad Q>0, P \leq \frac{-Q}{8} \text {; }
\end{aligned}
$$

where $P, Q$ and $R$ are given by (2.19).

Remark 1

- As $q \rightarrow 1$ Theorem 2.2 reduces to Theorem 1 in [10].

- As $q \rightarrow 1$ and $B_{1}=B_{2}=B_{3}=2$, Theorem 2.2 reduces to Theorem 3.1 in [7].

Definition 2.3. Let $\phi: U \rightarrow \mathrm{C}$ be analytic, and let $\phi(z)$ be given as in (2.1). The class $K^{j, k}(\phi)$ of $(j, k)$-convex symmetrical functions with respect to $\phi$ consists of functions f satisfying the subordination

$1+\frac{z f^{\prime \prime}(z)}{f_{j, k}^{\prime}(z)} \prec \varphi(z)$.

Theorem 2.4. Let the function $f \in K^{j, k}(\phi)$ be given by (1.1).

1.If $D_{1}, D_{2}$ and $D_{3}$ satisfy the conditions $\left(\frac{6\left(\psi_{3}+2 \psi_{2}\right)-16}{2}\right) D_{1}^{2} \psi_{1}^{2}+4\left|D_{2}\right| \psi_{1}^{2}-2 D_{1} \psi_{1}^{2} \leq 0$ and

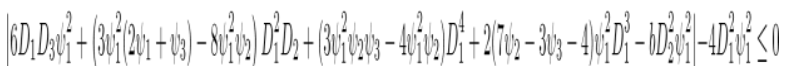

then the second Hankel determinant satisfies $\left|a_{2} a_{4}-a_{3}^{2}\right| \leq \frac{D_{1}^{2}}{36} \psi_{1}^{2}$.

2. If $D_{1}, D_{2}$ and $D_{3} \quad$ satisfy (2.10)

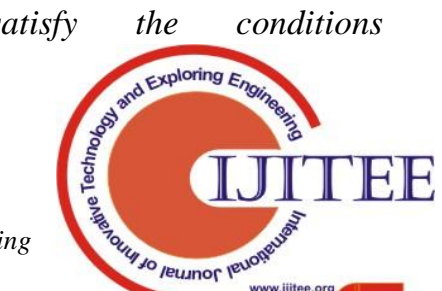


$\left(\frac{6\left(\psi_{3}+2 \psi_{2}\right)-16}{2}\right) D_{1}^{2} \psi_{1}^{2}+4\left|D_{2}\right| \psi_{1}^{2}-2 D_{1} \psi_{1}^{2} \geq 0$ and

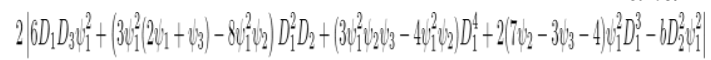

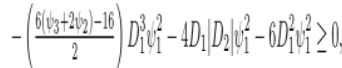

or the conditions

$\left(\frac{6\left(\psi_{3}+2 \psi_{2}\right)-16}{2}\right) D_{1}^{2} \psi_{1}^{2}+4\left|D_{2}\right| \psi_{1}^{2}-2 D_{1} \psi_{1}^{2} \leq 0$

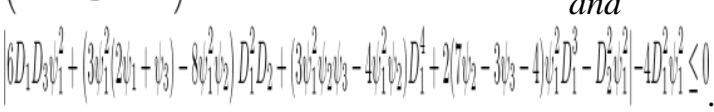

then the second Hankel determinant satisfies

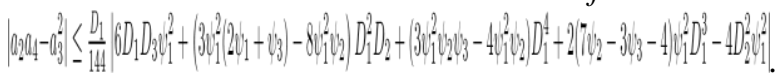

3. If $D_{1}, D_{2}$ and $D_{3}$ satisfy the conditions $\left(\frac{6\left(\psi_{3}+2 \psi_{2}\right)-16}{2}\right) D_{1}^{2} \psi_{1}^{2}+4\left|D_{2}\right| \psi_{1}^{2}-2 D_{1} \psi_{1}^{2}>0$ and

$2\left|6 D_{1} D_{3} \psi_{1}^{2}+\left(3 v_{1}^{2}\left(2 v_{1}+\psi_{3}\right)-8 v_{1}^{2} \psi_{2}\right) D_{1}^{2} D_{2}+\left(3 v_{1}^{2} \psi_{2} \psi_{3}-4 \psi_{1}^{2} v_{2}\right) D_{1}^{4}+2\left(7 v_{2}-3 \psi_{3}-4\right) \psi_{1}^{2} D_{1}^{3}-6 D_{2}^{2} \psi_{1}^{2}\right|$ $-\left(\frac{6\left(v_{3}+2 v_{2}\right)-16}{2}\right) D_{1}^{3} \psi_{1}^{2}-4 D_{1} \mid D_{2} \psi_{1}^{2}-6 D_{1}^{2} \psi_{1}^{2} \leq 0$

then the second Hankel determinant satisfies

$\left|a_{2} a_{4}-a_{3}^{2}\right| \leq \frac{D_{1}^{2} \psi_{1}^{2}}{576} \frac{\left(\begin{array}{r}16\left|6 D_{1} D_{3}+\gamma D_{1}^{2} D_{2}+\beta D_{1}^{4}+\delta D_{1}^{3}-4 D_{2}^{2}\right| \\ -12 \alpha D_{1}^{3}-48 D_{1}\left|D_{2}\right| \\ -36 D_{1}^{2}-\alpha D_{1}^{4}-8 \alpha D_{1}^{2}\left|D_{2}\right|-16 D_{2}^{2}\end{array}\right)}{\left|6 D_{1} D_{3}+\gamma D_{1}^{2} D_{2}+\beta D_{1}^{4}+\delta D_{1}^{3}-4 D_{2}^{2}\right|-\alpha D_{1}^{3}-4 D_{1}\left|D_{2}\right|-2 D_{1}}$

where

$$
\begin{aligned}
& \alpha=\left(\frac{6\left(\psi_{3}+2 \psi_{2}\right)-16}{2}\right), \quad \beta=\left(3 \psi_{2} \psi_{3}-4 \psi_{2}\right), \\
& \gamma=\left(3\left(2 \psi_{1}+\psi_{3}\right)-8 \psi_{2}\right),
\end{aligned}
$$

$\delta=2\left(7 \psi_{2}-3 \psi_{3}-4\right)$

Proof. Since $f \in K^{j, k}(\phi)$, there exists an analytic function $w$ with $w(0)=0$ and $|w(z)|<1$ in $\mathrm{U}$ such that

$$
1+\frac{z f^{\prime \prime}(z)}{f_{j, k}^{\prime}(z)}=\varphi(w(z))=1+\frac{1}{2} D_{1} c_{1} z+\left(\frac{1}{2} D_{1}\left(c_{2}-\frac{c_{1}^{2}}{2}\right)+\frac{1}{4} D_{2} c_{1}^{2}\right) z^{2}+\ldots
$$

so that

$$
\left(\frac{2 a_{2} z+6 a_{3} z^{2}+12 a_{4} z^{3}+20 a_{a} z^{4}+\ldots}{\psi_{1}+2 \psi_{2} a_{2} z+3 \psi_{3} a_{3} z^{2}+4 \psi_{4} a_{4} z^{3}+\ldots}\right)=1+\frac{1}{2} D_{1} c_{1} z+\left(\frac{1}{2} D_{1}\left(c_{2}-\frac{c_{1}^{2}}{2}\right)+\frac{1}{4} D_{2} c_{1}^{2}\right) z^{2}+\ldots
$$

which implies the equality

$$
\begin{aligned}
& \left.2 a_{2} z+6 a_{3} z^{2}+12 a_{4} z^{3}+2 a_{a_{3}} z^{4}+\ldots\right)=\left(1+\frac{1}{2} D_{1} c_{1} u_{1}\right) z+\left(a_{2} D_{1} c_{1} u_{2}+u_{1}\left(\frac{1}{2}-D_{1}\left(c_{2}-\frac{c_{1}^{2}}{2}\right)+\frac{1}{4} D_{2} c_{1}\right)\right) z^{2} \\
& +\left(a_{2}^{3} D_{1} D_{1} v_{3}+v_{2} a_{2}\left(\frac{1}{2}-D_{1}\left(C_{2}-\frac{C_{1}^{2}}{2}\right)+\frac{1}{4} D_{2} C_{1}^{2}\right)+v_{1}\left(D_{1}\left(\frac{C_{3}}{2}-\frac{C_{1} C_{2}}{2}+\frac{C_{1}^{3}}{8}\right)+D_{2} C_{1}\left(\frac{C_{2}}{2}-\frac{C_{1}^{2}}{4}\right)+\frac{D_{3} C_{1}^{3}}{8}\right)\right) z^{3}+\ldots
\end{aligned}
$$

Equating the coefficients on both sides we have

$a_{2}=\frac{D_{1} c_{1} \psi_{1}}{4}$

$$
\begin{aligned}
a_{3}= & \frac{1}{24}\left(\left(D_{1}^{2} \psi_{2}-D_{1}+D_{2}\right) c_{1}^{2} \psi_{1}+2 D_{1} c_{2} \psi_{1}\right), \\
a_{4}=\frac{\psi_{1}}{192}\left[\left(-4 D_{2}+2 D_{1}+\right.\right. & \left.D_{1}^{3} \psi_{2} \psi_{3}-3 D_{1}^{2} \psi_{3}+\left(2 \psi_{1}+\psi_{3}\right) D_{1} D_{2}+2 D_{3}\right) c_{1}^{3} \\
& \left.+2\left(\left(\psi_{3}+2 \psi_{2}\right) D_{1}^{2}-4 D_{1}+4 D_{2}\right) c_{1} c_{2}+8 D_{1} c_{3}\right] .
\end{aligned}
$$

Therefore

$$
\begin{aligned}
a_{2} a_{4}-a_{3}^{2}=\frac{D_{1} \psi_{1}^{2}}{768}\left[c _ { 1 } ^ { 4 } \left(\frac{-4}{3} D_{2}+\frac{2}{3} D_{1}\right.\right. & \left.+\frac{D_{1}^{3}}{3} \beta-\frac{D_{1}^{2}}{3}\left(9 \psi_{3}-8 \psi_{2}\right)+\frac{D_{1} D_{2}}{3} \gamma+2 D_{3}-\frac{4 D_{2}^{2}}{3 D_{1}}\right) \\
& \left.+\frac{2 c_{1}^{2} c_{2}}{3}\left(\alpha D_{1}^{2}-4 D_{1}+4 D_{2}\right)+8 D_{1} c_{1} c_{3}-\frac{16}{3} D_{1} c_{2}^{2}\right] .
\end{aligned}
$$

where

$\alpha, \beta, \gamma$ are given by (2.13) By writing

$$
d_{1}=8 D_{1}, \quad d_{2}=\frac{2}{3}\left(2 D_{1}^{2}-4 D_{1}+D_{2}\right), \quad d_{3}=\stackrel{\left[-16 D_{1}\right.}{3},
$$

$d_{4}=\left(\frac{-4}{3} D_{2}+\frac{2}{3} D_{1}+X D_{1}^{3}-\left(9 \psi_{3}-8 \psi_{2}\right) \frac{D_{1}^{2}}{3}+\frac{Y}{3} D_{1} D_{2}+2 D_{3}-4 \frac{D_{2}^{2}}{3 D_{1}}\right)$

$T=\frac{D_{1}}{768}$.

we have

$$
\left|a_{2} a_{4}-a_{3}^{2}\right|=T\left|d_{1} c_{1} c_{3}+d_{2} c_{1}^{2} c_{2}+d_{3} c_{2}^{2}+d_{4} c_{1}^{4}\right|
$$

Since the function $p\left(e^{i \theta} z\right)(\theta \in \mathrm{R})$ is in the class $\mathrm{P}$ for any $p \in$ $\mathrm{P}$, there is no loss of generality in assuming $c_{1}>0$. Write $c_{1}=$ $c, c \in[0,2]$. Substituting the values of $c_{2}$ and $c_{3}$ respectively from (1.5) and (1.6) in (2.16), we obtain $\left|a_{2} a_{4}-a_{3}^{2}\right|=\frac{T}{4} \mid c^{4}\left(d_{1}+2 d_{2}+d_{3}+4 d_{4}\right)+2 x c^{2}\left(4-c^{2}\right)\left(d_{1}+d_{2}+d_{3}\right)$

$$
+\left(4-c^{2}\right) x^{2}\left(-d_{1} c^{2}+d_{3}\left(4-c^{2}\right)\right)+2 d_{1} c\left(4-c^{2}\right)\left(1-|x|^{2} z\right) \mid .
$$

Replacing $|x|$ by $\mu$ and substituting the values of $d_{1}, d_{2}, d_{3}$ and $d_{4}$ from (2.15) yield

$$
\begin{array}{r}
\left|a_{2} a_{4}-a_{3}^{2}\right| \leq \frac{T\left(\psi_{1}^{2}\right)}{4}\left[c^{4}\left|\frac{4}{3} \beta D_{1}^{3}+\frac{4}{3} \gamma D_{1} D_{2}+8 D_{3}-\frac{16 D_{2}^{2}}{3 D_{1}}+\frac{4}{3} \delta B_{1}^{2}\right|+\frac{4 \mu c^{2}\left(4-c^{2}\right)}{3} a D_{1}^{2}+\frac{8}{3}\left|D_{2}\right|\right. \\
\left.+\mu^{2}\left(4-c^{2}\right)\left(\frac{8}{3} D_{1} c^{2}+\frac{64}{3} D_{1}\right)+16 D_{1} c\left(4-c^{2}\right)\left(1-\mu^{2}\right)\right] . \\
=T\left[\frac{c^{4}}{3}\left|\beta D_{1}^{3}+\gamma D_{1} D_{2}+6 D_{3}-4\left(D_{2}^{2} / D_{1}\right)+\delta D_{1}^{2}\right|+4 D_{1} c\left(4-c^{2}\right)+\frac{\mu c^{2}\left(4-c^{2}\right)}{3}\left(\alpha D_{1}^{2}+4\left|D_{2}\right|\right)+\frac{2 D_{1}}{3}\right. \\
\left.\frac{\mu^{2}\left(4-c^{2}\right) D_{1}}{3}((c-2)(c-4))\right]
\end{array}
$$

$$
\equiv F(c, \mu), \quad(2.17)
$$

where $\alpha, \beta$, $\gamma$ and $\delta$ are given by (2.13).

Again, differentiating $F(c, \mu)$ in (2.17) partially with respect to $\mu$ yields

$$
\frac{\partial F}{\partial \mu}=T\left[\frac{c^{2}}{3}\left(4-c^{2}\right)\left(\alpha D_{1}^{2}+4\left|D_{2}\right|\right)+\frac{2 \mu\left(4-c^{2}\right) D_{1}}{3}((c-2)(c-4))\right] .
$$

Then, for $0<\mu<1,0<q<1$ and for any fixed $c$ with $0<c$ $<2$, it is clear from (2.18) that $\frac{\partial F}{\partial \mu}>0$, that is, $F(c, \mu)$ is an increasing function of $\mu$. Hence, for fixed $c \in[0,2]$, the maximum of $F(c, \mu)$ occurs at $\mu=1$, and $\max F(c, \mu)=F(c, 1) \equiv G(c)$.

Also note that

$$
\begin{array}{r}
G(c)=T\left[\frac{c^{4}}{3}\left(\left|\beta D_{1}^{3}+\gamma D_{1} D_{2}+6 D_{3}-4\left(D_{2}^{2} / D_{1}\right)+\delta D_{1}^{2}\right|-\alpha D_{1}^{2}-4\left|D_{2}\right|-2 D_{1}\right)\right. \\
\left.+\frac{4 c^{2}}{3}\left(\alpha D_{1}^{2}+4\left|D_{2}\right|-2 D_{1}\right)+\frac{64}{3} D_{1}\right],
\end{array}
$$

where $\alpha, \beta, \gamma$ and $\delta$ are given by (2.13). Let

$P=\frac{1}{3}\left(\left|\beta D_{1}^{3}+\gamma D_{1} D_{2}+6 D_{3}-4\left(D_{2}^{2} / D_{1}\right)+\delta D_{1}^{2}\right|-\alpha D_{1}^{2}-4\left|D_{2}\right|-2 D_{1}\right)$

$Q=\frac{4}{3}\left(\alpha D_{1}^{2}+4\left|D_{2}\right|-2 D_{1}\right), \quad R=\frac{64}{3} D_{1}$.

(2.19)

By using (2.12) we get

$$
\begin{aligned}
& \left\lceil, \quad Q \leq 0, P \leq \frac{-Q}{4} ;\right. \\
& \left|a_{2} a_{4}-a_{3}^{2}\right| \leq \frac{B_{1}}{768}\left\{16 X+4 Y+Z, Q \geq 0, X \geq \frac{-Q}{8} \text { or } Q \leq 0, P \geq \frac{-Q}{4}\right. \text {; } \\
& \frac{4 P R-Q^{2}}{4 P}, \quad Q>0, P \leq \frac{-Q}{8} ;
\end{aligned}
$$

Where, $X, Y$ and $Z$ are given by (2.19).

Remark 2: 
- As $q \rightarrow 1$ Theorem 2.4 reduces to Theorem 2 in [10].

- As $q \rightarrow 1$ for the choice $\varphi(z)=((1+z) /(1-z))$, Theorem 2.4 reduces to Theorem

3.2 in [7].

\section{CONCLUSION}

We study the estimates for the Second Hankel determinant of analytic functions. Our class includes $(j, k)$-convex, $(j, k)$-starlike functions and Ma-Minda starlike and convex functions..

\section{REFERENCES}

1. P. L. Duren, Univalent Functions, Grundlehren Math. Wiss., Springer, New York, 259(1983)

2. R. Ehrenborg, The Hankel determinant of exponential polynomials, Amer. Math. Mont., 107(6) (2000), 557-560.

3. M. Fekete and G. Szeg"o , Eine Bermerkung U“ber ungerade schlichte Funktionen, J. London Math. Soc., 1(2)(1933), 85-89.

4. U. Grenander, G. Szeg"o, Toeplitz Forms and Their Applications, California Monographs Math. Sci. University of California Press, Berkeley, (1958)

5. W. K. Hayman, On the second Hankel determinant of mean univalent functions, Proc. London Math. Soc., 3(18)(1968), 77-94.

6. F. H. Jackson, On q-functions and a certain difference operator, Trans. Royal Soc. Edinburgh, 46(1909), 253-281.

7. A. Janteng, S. Halim and M. Darus, Hankel determinant forstarlike and convex functions, Int. J. Math. Anal. (Ruse), 1(13-16)(2007), 619-625.

8. S. Kanas, W. Wisniowska, Conic regions and k-uniform convexity, J. Comput. Appl. Math., 105(1999), 327-336.

9. S. Kanas, W. Wisniowska, Conic domains and starlike functions, Rev. Roumaine Math. Pures Appl., 45(2000), 647-657.

10. L. S. Keong, V. Ravichandran, and S. Supramaniam. Bounds for the second Hankel determinant of certain univalent functions, arXiv preprint arXiv:1303.0134 (2013).

11. P. Liczberski and J. Polubinski, On (j,k)-symmtrical functions, Mathematica Bohemica,120 (1995), 13-25.

12. K. I. Noor and S. A. Al-Bany, On Bazilevic functions, Int. J. Math. Math. Sci., 1(10)(1987), 79-88.

13. J. W.Noonan, and D. K. Thomas. On the second Hankel determinant of areally mean univalent functions, Transactions of the Amer. Math. Soci. 223(1976) 337-346.

14. C. Pommerenke, On the coefficients and Hankel determinants of univalent functions, J. London Math.Soc.,

15. 41(1966), 111-122.

16. C. Pommerenke, On the Hankel determinants of univalent functions, Math., 14(1967), 108-112.

17. C. Pommerenke, G. Jensen, Univalent functions, Vandenhoeck und Ruprecht, 25 (1975).

18. F. Rønning, Uniformly convex functions and a corresponding class of starlike functions, Proc. Am. Math. Soc., 118(1)(1993), 189-196.

19. H. Shamsan, F. S. Alsarari, and S. Latha, Bounds for the Second g-Hankel Determinant of Certain

20. Univalent Functions, Int. J. Math. And Appl., 6(1-D)(2018), 809-817.

21. T. Thulasiram, K. Suchithra and R. Sattanathan, Second Hankel determinant for some subclasses of analytic functions, Int. J. Math. Sci Appl., 2(2012), 653-661.

22. D. Vamshee Krishna and T. Ramreddy, Coefficient inequality for certain subclasses of analytic functions, New Zealand J. Math., 42(2012), 217-228.

\section{AUTHORS PROFILE}

RENUKA DEVI K Department of Mathematics, Yuvaraja's College, University of Mysore,Mysuru-570 005, INDIA.

HAMID SHAMSAN Department of Mathematics, Yuvaraja's College, University of Mysore,Mysuru-570 005, INDIA.

S. LATHA Department of Mathematics, Yuvaraja's College, University of Mysore,Mysuru-570 005, INDIA. 